\section{Encuesta de opinión pública sobre reproducción humana y usos de tecnología de reproducción asistida en habitantes de Santiago, Chile}

\section{Opinion survey on the use of assisted reproductive technologies applied to inhabitants of Santiago, Chile}

Background: Advances in reproductive medicine and the use of assisted reproductive technologies (ART) have a great impact in the lives of people and the conformation of their families. Aim: To report the results of an opinion survey applied to inhabitants of Santiago, Chile about the use of ART to conceive. Material and Methods: A survey was designed and applied to a random representative sample of 1.500 people between the ages of 18 and 65 in the 34 municipalities of Santiago. Results: Eighty eight percent of respondents support the use of medical assistance to conceive children. Wide approval exists for the use of in vitro fertilization (IVF) by heterosexual couples, even when not married (85.9\%) and by single women (70.4\%), by both male and female participants of every socioeconomic level, age group and religious affiliation. Support decreases significantly when the use of IVF is considered for post-menopausal women (35.1\%) and by same-sex couples (26.6\%). Conclusions: Results of this survey indicate that the majority of inhabitants in Santiago favor the use of ART, including IVF. This support decreases significantly for elderly women and homosexual couples.

(Rev Med Chile 2013; 141: 853-860).

Key words: Fertilization in vitro; Health surveys; Infertility.

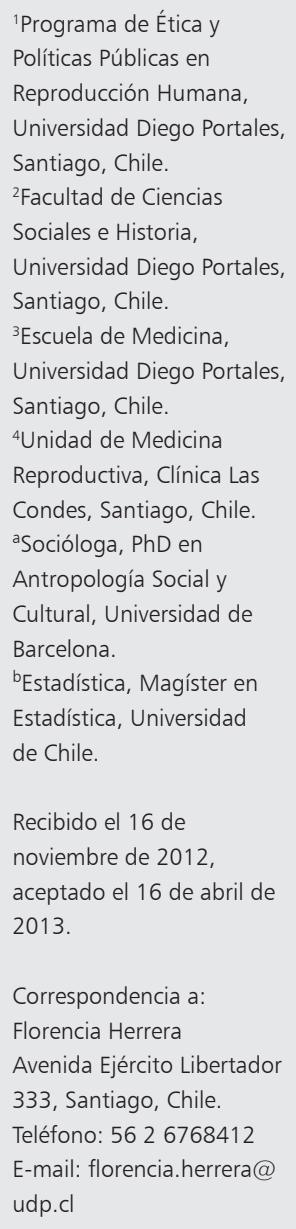

Correspondencia a: Florencia Herrera Avenida Ejército Libertador 333, Santiago, Chile. Teléfono: 5626768412 E-mail: florencia.herrera@ udp.cl

L a creciente capacidad de intervenir en los inicios de la vida humana mediante el uso de técnicas de reproducción asistida (TRA) ha permitido que parejas infértiles, mujeres mayores, parejas homosexuales y personas sin pareja accedan a la posibilidad de concebir hijos. Estas técnicas han puesto en el centro de la discusión los derechos a concebir que la sociedad le otorga a las personas y los deberes que acompañan estos derechos.

Se entiende por TRA todos los tratamientos o procedimientos que incluyen la manipulación de ovocitos, espermatozoides o embriones humanos para el establecimiento de un embarazo. Esto incluye la fecundación in vitro (FIV) y la transferencia de embriones; la transferencia intratubárica de gametos, zigotos, o embriones; la criopreservación de ovocitos y embriones; la donación de ovocitos y embriones y el útero surrogado. Las TRA no incluyen la inseminación artificial usando espermatozoides de la pareja o de un donante ${ }^{1}$.

La utilización de estas técnicas aumenta a una tasa anual de aproximadamente $10 \%$, independientemente de la tasa de crecimiento poblacional del país ${ }^{2}$, reflejando que la infertilidad es vivida como un problema de las personas, independien- 
temente de consideraciones demográficas, sociales o económicas.

Se estima que la infertilidad afecta aproximadamente al $10 \%$ de las parejas en edad reproductiva ${ }^{3}$. La incapacidad para tener hijos repercute en el bienestar de las personas y a su núcleo familiar, especialmente a las mujeres, ya que son las que sienten mayor presión social para convertirse en madres, suelen ser consideradas culpables por los fracasos reproductivos y son sus cuerpos los que deben soportar las intervenciones médicas y sus consecuencias ${ }^{4,5}$. En parte, este efecto negativo en las personas y en su entorno familiar es lo que llevó a la Organización Mundial de la Salud (OMS) junto a otras organizaciones, a definir la infertilidad como una enfermedad ${ }^{1}$.

De los cerca de 5.000.000 de personas nacidas en el mundo producto de las nuevas tecnologías reproductivas ${ }^{6}$, aproximadamente 100.000 han nacido en Latinoamérica ${ }^{7}$ y 5.500 en Chile ${ }^{8}$. A pesar de la gran cantidad de niños nacidos mediante estos tratamientos médicos, existen pocos estudios que indaguen la percepción pública sobre estas tecnologías reproductivas. Las investigaciones suelen centrarse en las experiencias y opiniones de pacientes y profesionales de la salud ${ }^{9-12}$ o de poblaciones específicas, como mujeres u hombres en edad reproductiva, mujeres infértiles, o estudiantes de psicología ${ }^{13-18}$. Asimismo, las encuestas realizadas tienden a centrarse en temas particulares, como la donación de gametos ${ }^{19-22} \mathrm{o}$ la gestación sustituta ${ }^{14,23,24}$. No existen estudios en Latinoamérica que examinen los conocimientos y opiniones de la población general en relación al uso de TRA para concebir.

El objetivo del presente trabajo es conocer la opinión de los habitantes de Santiago, Chile, respecto del uso de TRA. En este primer artículo, se presenta la posición de los encuestados respecto al uso de la asistencia médica para concebir hijos; la fecundación in vitro (FIV); el uso de FIV por parte de parejas no casadas, mujeres solas, parejas del mismo sexo y mujeres después de la menopausia; el financiamiento de las TRA por parte del Estado y, finalmente, la reproducción con donación de gametos.

\section{Material y Método}

Para realizar la investigación se diseñó una encuesta que fue aplicada entre el 30 de septiembre y el 23 de octubre de 2011 a una muestra aleatoria representativa de la población de Santiago de 1.500 personas.

Se incluyeron 114 preguntas de conocimiento general de fertilidad humana y opiniones sobre reproducción médicamente asistida, posición respecto a quiénes deben tener acceso a TRA, opinión sobre el financiamiento de estos tratamientos y posición respecto a la donación de gametos. El cuestionario fue diseñado por un equipo multidisciplinario de especialistas y luego fue revisado por otros investigadores y académicos del área. Con el fin de validar las preguntas, establecer la duración de la aplicación del cuestionario, y asegurar que éste fuera comprensible para los encuestados, se realizaron dos pre-tests, el primero a 8 personas y el segundo a 30, representativas de los potenciales encuestados en cuanto a edad, sexo y grupo socioeconómico. En base a esta información se definió el cuestionario final.

La encuesta fue aplicada por 54 encuestadores previamente capacitados. Para asegurar que todos los encuestados manejaran un nivel de información que les permitiera contestar las preguntas, se introdujeron párrafos explicativos de temas como: en qué consisten los TRA, fecundación in vitro y donación de gametos. Estos párrafos fueron redactados de forma clara y con lenguaje sencillo, evitando inducir la respuesta del encuestado. Las definiciones utilizadas en la encuesta fueron extraídas de la traducción oficial al español del glosario de terminologías publicadas en 2009 por el International Committe for Monitoring Assisted Reproductive Technology y la OMS ${ }^{1}$.

El estudio fue aprobado por el Comité de Ética de Investigación de la Universidad Diego Portales (UDP). Los participantes leyeron y firmaron un consentimiento informado donde se establecía que su participación en el estudio sería voluntaria y anónima.

\section{Características de la muestra}

La muestra fue representativa de los habitantes de 34 comunas de Santiago (Provincia de Santiago más Puente Alto y San Bernardo), con edades entre 18 y 65 años. El marco muestral utilizado fue el Censo del año 2002 actualizado (Disponible en: http://www.ine.cl/canales/chile_estadistico/familias/demograficas_vitales.php).

La muestra fue probabilística en todas sus etapas, su diseño fue estratificado por comunas 
y luego se seleccionaron conglomerados (manzanas) en cada comuna, con selección aleatoria de viviendas. En cada vivienda se aplicó una tabla de sorteo aleatorio única para cada entrevista, la que determinó la persona a ser entrevistada. La muestra fue distribuida en 250 conglomerados, realizándose 6 entrevistas por conglomerado.

Las entrevistas realizadas a sujetos sorteados en primera instancia, alcanzaron al $62,7 \%$. Los reemplazos normales alcanzaron al 25,5\%, hubo $7,7 \%$ reemplazos por no vivir un sujeto de 18 a 65 en el hogar y $4,1 \%$ de rechazos a la carta de consentimiento.

\section{Tamaño de la muestra}

Para el cálculo del tamaño teórico de una muestra representativa mediante muestreo aleatorio se utilizó el supuesto de máxima varianza ${ }^{25,26}$, y se calcularon tamaños de muestras según sexo, tramos de edad y grupo socioeconómico, lo que permitió realizar inferencias estadísticas para estos grupos. El cálculo del tamaño muestral fue de 1.500 personas. El error muestral si la muestra fuera completamente aleatoria es de $2,5 \%$, con un nivel de confianza de $95 \%$.

Este diseño muestral permite realizar inferencias estadísticas según sexo, grupos de edad y grupos socioeconómicos (GSE). La conformación final de la muestra se describe en la Tabla 1.

\section{Análisis estadístico}

Se construyeron tablas de contingencia, y a cada una de ellas se le aplicó una prueba de independencia utilizando el estadístico "chi-cuadrado" para determinar relaciones entre variables sociodemográficas, incluyendo religión, y respuestas de los individuos. Respecto a la religión, se hizo la distinción entre católicos religiosos (personas religiosas y cercanas a la religión católica) y católicos no religiosos (no se consideran practicantes de la religión católica, pero sí cercanos a ella). Se consideró estadísticamente significativo un $\mathrm{p}<0,05$.

\section{Resultados}

Los resultados globales se presentan en forma de figuras o tablas, mientras que la información que resulta al analizar los datos por edad, sexo, grupo socio-económico (GSE) y religión es descrita en el texto.

\section{Aceptabilidad en el uso de TRA}

Como se muestra en la Figura 1, 89,2\% de los encuestados estaba de acuerdo con que toda persona tiene derecho a hacer uso de los avances de la medicina y $88,1 \%$ apoyaba el uso de la asistencia médica para concebir hijos. Sólo 19,6\% pensaba que las personas que no pueden concebir hijos de manera espontánea no debiesen tenerlos, rechazo que fue menor en los más jóvenes $(14,4 \%)$ y en el GSE medio alto $(14,8 \%)$.

La mayoría de los encuestados $(71,8 \%)$ apoyaba el uso de FIV (Figura 2), observándose mayor apoyo en las mujeres $(73,5 \%)$, en las personas menores de 44 años (76\%), y en el GSE más alto (77,9\%). $\mathrm{Al}$ analizar las respuestas según religión, el apoyo fluctuó entre $64,1 \%$ en los encuestados evangélicos y $78,2 \%$ en los católicos no religiosos $(p<0,05)$.

La aceptación al uso de FIV disminuyó a 58,7\% cuando se pregunta a los encuestados si personalmente considerarían su uso en caso de no poder tener hijos.

\section{Quienes deben tener acceso a TRA}

Tal como se muestra en la Figura 3, hubo amplio apoyo para el acceso a TRA por parte de parejas heterosexuales, aun cuando no estén casadas $(85,9 \%)$, y por parte de mujeres solas $(70,4 \%)$. Este apoyo fue transversal según sexo y en los distintos GSE, siendo menor en las personas evangélicas $(62,6 \%, p<0,05)$. La aprobación disminuyó significativamente cuando se trata del uso de TRA

Tabla 1. Estructura de la muestra

\begin{tabular}{|lcrc|}
\hline \multicolumn{1}{|c}{$\mathbf{n}$} & \multicolumn{1}{c}{$\%$} & Error muestral \\
Sexo & & & \\
Hombre & 749 & 49,9 & $3,6 \%$ \\
Mujer & 751 & 50,1 & $3,6 \%$ \\
Total & 1.500 & 100,0 & $2,5 \%$ \\
Edad & & & \\
18 a 29 años & 382 & 25,5 & $5,0 \%$ \\
30 a 44 años & 468 & 31,2 & $4,5 \%$ \\
45 a 65 años & 650 & 43,3 & $3,8 \%$ \\
Total & 1.500 & 100,0 & $2,5 \%$ \\
Grupo socioeconómico (GSE) & \\
Medio-alto & 420 & 28,0 & $4,8 \%$ \\
Medio & 600 & 40,0 & $4,0 \%$ \\
Bajo & 480 & 32,0 & $4,5 \%$ \\
Total & 1.500 & 100,0 & $2,5 \%$ \\
\hline
\end{tabular}




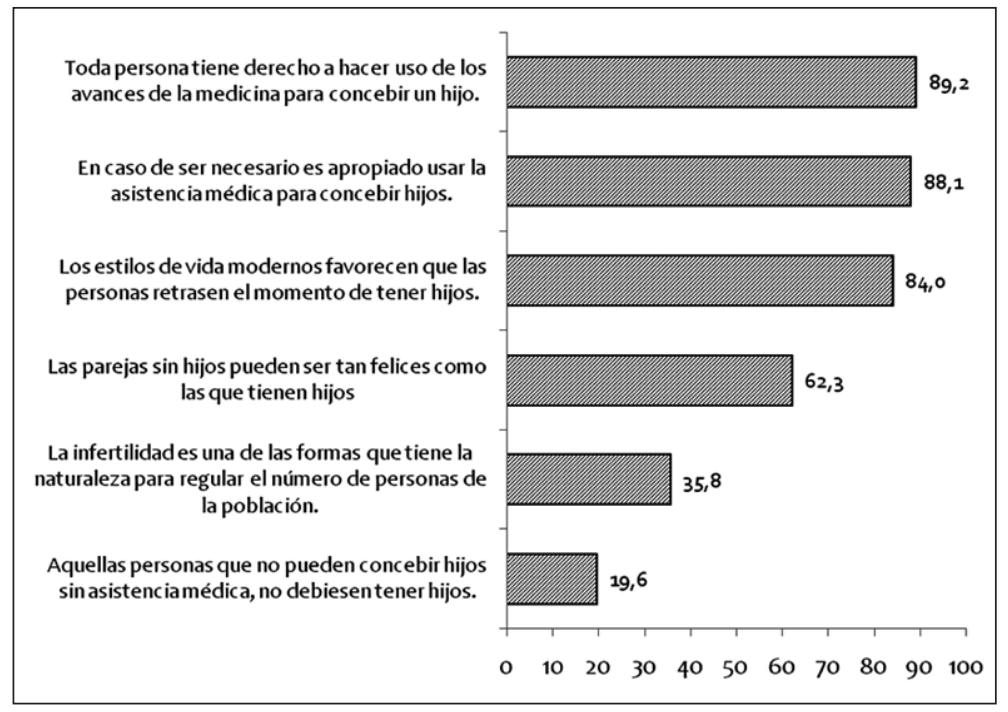

Figura 1. Acuerdo o desacuerdo con afirmaciones sobre reproducción y asistencia médica. \% Menciones "De acuerdo".

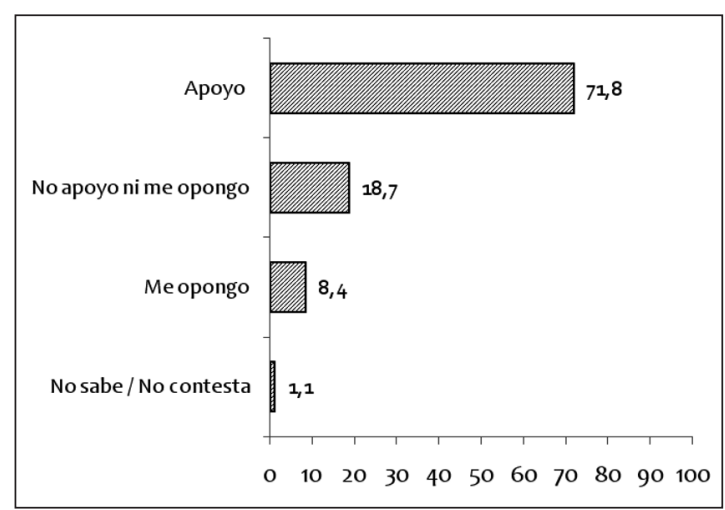

Figura 2. Apoyo al uso de fecundación in vitro. \% Menciones "Apoyo". por parte de mujeres post menopáusicas $(35,1 \%)$ y por parejas homosexuales $(26,6 \%)$, siendo algo superior en los encuestados de 18 a 29 años (35\%) $y$ en personas que no profesan religión $(40,9 \%)$.

\section{Fuente de financiamiento de TRA}

Cuarenta y ocho por ciento de los encuestados consideró que el Estado debería financiar los tratamientos sólo a quienes no pueden costearlos, mientras que $43,5 \%$ opinó que este financiamiento debe ser otorgado a todos los individuos por igual (Figura 4). Cincuenta y cuatro por ciento de las personas de GSE bajo se inclinaron por un financiamiento para todos, mientras que $57,4 \%$ de las

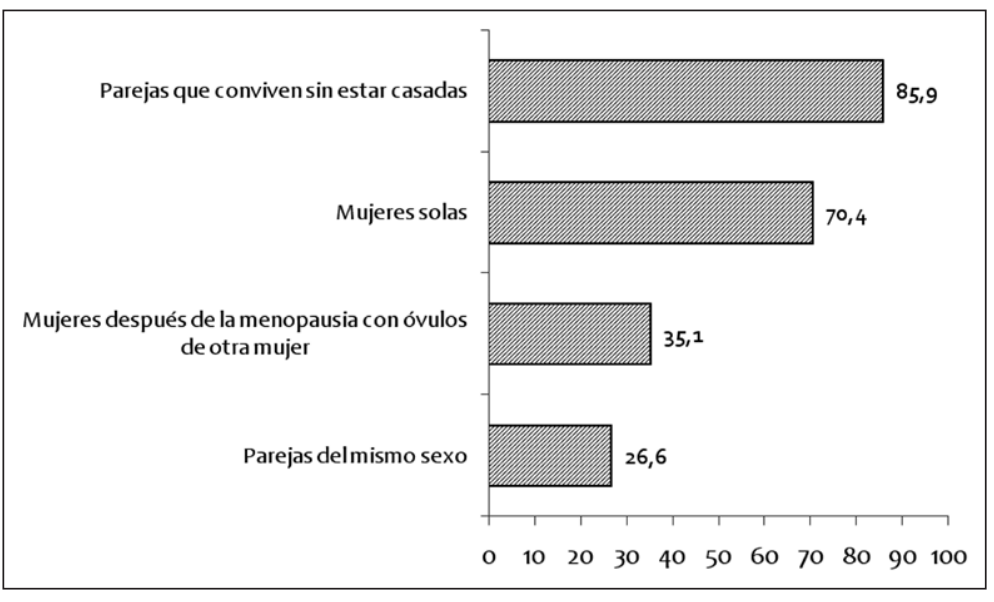

Figura 3. Apoyo al uso de fecundación in vitro por parte de distintas estructuras familiares. \% Menciones "Apoyo". 
de GSE medio-alto prefirieron financiar sólo a quienes no pueden pagar.

Los encuestados mayoritariamente desaprobaron el financiamiento estatal para las mujeres mayores de 50 años $(62,9 \%)$ y para parejas lesbianas $(66,6 \%)$.

\section{Uso de gametos de donantes para concebir}

La mayoría de los encuestados estuvo de acuerdo con la donación de gametos para concebir (Figura 5), ya sea para ser utilizados en parejas infértiles (79\%) o en mujeres solas (71,5\%). Hubo mayor acuerdo con el uso de gametos de donante entre las personas de 18 a 29 años, respecto de personas mayores $(\mathrm{p}<0,001)$. Concordante con los datos anteriores, este alto nivel de aceptación disminuyó cuando las receptoras son una pareja lesbiana (32\%) o una mujer menopáusica $(35,6 \%)$.

La mayoría de los encuestados consideraba

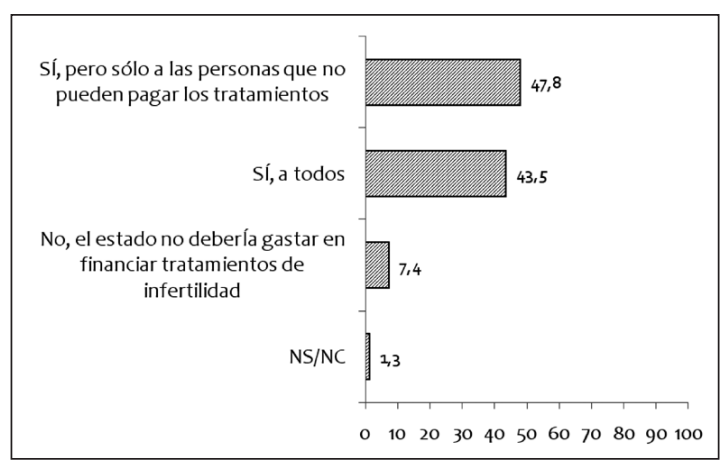

Figura 4. Acuerdo con financiamiento de TRA por parte del Estado. \% Menciones "De acuerdo". que la donación de gametos debiese ser gratuita (72\%), anónima (68\%), y que los donantes no debiesen tener ninguna obligación hacia los niños nacidos $(78,2 \%)$. En ninguna de estas respuestas se observaron diferencias en consideración a si el gameto donado era masculino o femenino.

Sesenta y siete por ciento de los encuestados respondió que sí donaría sus gametos para que una pareja infértil tenga un hijo (50,2\% lo haría en forma anónima y 16,7\% incluso si su identidad es dada a conocer). Sólo 27,5\% señaló no estar dispuesto a ser donante de gametos. Asimismo, 57,2\% de las personas encuestadas recibiría gametos de un/una donante, $(23,5 \%$ preferiría recibirlo de un pariente o amigo y $33,7 \%$ de un desconocido). Treinta y uno coma seis por ciento señaló que no aceptaría tener hijos con gametos donados.

\section{Discusión}

Los resultados de este estudio muestran que la mayoría de los habitantes del Gran Santiago está a favor del uso de TRA para el tratamiento de la infertilidad. Este apoyo se observó tanto en hombres como en mujeres, en todo nivel socioeconómico, $\mathrm{y}$ en todo el espectro etario y religioso.

Estos resultados son similares a los obtenidos recientemente en Australia, donde el apoyo a la FIV para parejas infértiles casadas alcanza $91 \%{ }^{27}$ y muestran mayor apoyo que el observado en población alemana, donde sólo 50\% de los encuestados estuvo de acuerdo con que parejas infértiles usaran todos los avances biomédicos para tener hijos biológicos ${ }^{28}$.

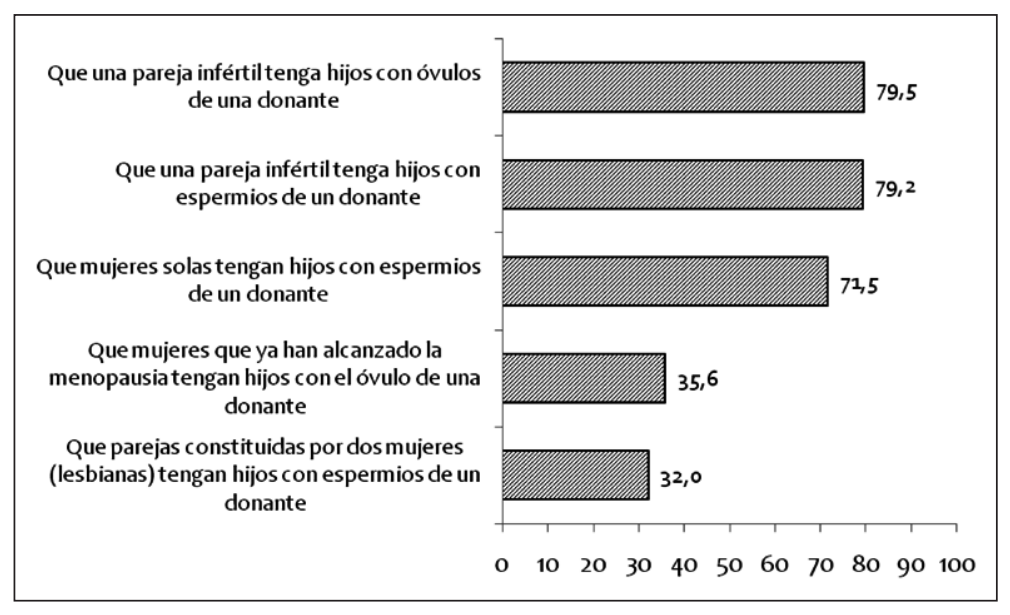

Figura 5. Acuerdo ante afirmaciones sobre donación de gametos. \% Menciones "De acuerdo". 
Es interesante señalar que existe amplia aceptación para el uso de gametos donados. Este apoyo supera lo descrito en población alemana, donde sólo 50\% de los encuestados aprueba la ovodonación, principalmente por razones médicas ${ }^{29}$. Otro aspecto a destacar se refiere a la nula diferencia que se hace respecto de la donación de gametos femeninos o masculinos.

Respecto de la estructura familiar deseable para acceder a estos tratamientos, la mayoría no está de acuerdo con el uso de estas tecnologías por parte de mujeres menopáusicas o parejas homosexuales. Estos resultados son consistentes con los resultados de la Encuesta Nacional de Opinión Pública 2012 de la UDP, donde sólo 30\% de los encuestados considera que una pareja homosexual debería ejercer su derecho a adoptar hijos (Disponible en: http://www.encuesta.udp.cl/wp-content/ uploads/2012/09/ChilenosyHomosexualidad 1. pdf). En el seguimiento de tres décadas realizado en Australia acerca de la FIV, se observa que el acuerdo con la maternidad lésbica a través de donación de gametos ha subido significativamente a lo largo de los años, alcanzando 60,8\% el año $2011^{27}$. En Chile no existen encuestas que permitan medir un lapso de tiempo tan amplio, pero los datos de las últimas tres Encuestas Nacionales de Opinión Pública de la UDP (2010, 2011 y 2012), muestran una tendencia a una mayor aceptación a la parentalidad homosexual.

Es interesante señalar el amplio rechazo al uso de TRA por mujeres postmenopáusicas. Debido a que el presente estudio no exploró respecto de las causas de este rechazo, no es posible determinar si éste se relaciona con la percepción de mayor riesgo materno asociado al procedimiento, consideración a un eventual detrimento de la calidad de vida de un hijo nacido de madre mayor ${ }^{30}$, o si se relaciona con la forma en que se distribuyen recursos escasos, en los cuales el acceso a TRA por parte de mujeres mayores puede competir con el acceso por parte de mujeres más jóvenes.

Respecto del financiamiento de las TRA, nuestros datos muestran que los habitantes de Santiago consideran que los tratamientos de fertilización asistida, incluso en mujeres solas, debiesen ser financiados por el Estado, al menos en aquellos casos en los cuales las personas no los pueden financiar. Esto contrasta con la realidad que viven las parejas infértiles en Chile, que reciben escaso apoyo económico por parte de los sistemas de salud. Así, las ISAPRES no dan cobertura a los procedimientos diagnósticos ni terapéuticos destinados al manejo de la infertilidad, mientras que el Fondo Nacional de Salud (FONASA) financia un porcentaje muy bajo de los ciclos terapéuticos realizados en Chile. Esto es coincidente con los datos en otros países, donde la mayoría de los encuestados considera que los TRA deben tener financiamiento estatal o deben ser reembolsables por el sistema de salud correspondiente $27,31,32$.

La aceptación a las TRA disminuye cuando se pregunta a la persona si personalmente utilizaría estas técnicas. Es decir, algunas personas estiman correcto que las TRA estén disponibles para las personas que necesiten acudir a ellas, aunque ellas mismas no estén dispuestas a someterse a estos tratamientos, ni aceptan ser donantes. Esto sugiere que los encuestados consideran el acceso a estas tecnologías como un asunto de justicia, independiente de si ellos son los afectados y, por otra parte, respetan la autonomía de las personas para tomar sus propias decisiones reproductivas, aún cuando estas decisiones vayan en contra de lo que ellas/os aceptan como opción reproductiva para ellas/os mismos.

En conclusión, los resultados del presente estudio muestran que los habitantes de Santiago apoyan el uso de TRA y FIV para que parejas heterosexuales y mujeres solas puedan tener hijos, manifestando desacuerdo en el uso de estas técnicas en mujeres postmenopáusicas y parejas homosexuales. Esta investigación contribuye -con evidencia cuantitativa sobre la opinión de los habitantes del Gran Santiago en relación al uso de la ciencia y la tecnología para concebir hijos- a los debates existentes actualmente en materia de acceso a los avances en medicina reproductiva.

Agradecimientos: Se agradece el apoyo del Fondo Nacional para la Ciencia y la Tecnología (FONDECYT) (Proyecto Fondecyt No 11110287) y del Programa de Ética y Políticas Públicas en Reproducción Humana (PREPRE) de la Universidad Diego Portales. Asimismo, se agradece la valiosa ayuda de Nincen Figueroa, ayudante de investigación, en el procesamiento de los datos.

\section{Referencias}

1. Zegers-Hochschild F, Adamson GD, De Mouzon J, Ishihara O, Mansour R, Nygren K, et al. International Com- 
mittee for Monitoring Assisted Reproductive Technology (ICMART) and the World Health Organization (WHO) revised glossary of ART terminology. Fertil. Steril. 2009; 92 (5): 1520-4. Hum. Reprod 2009; 24 (11): 2683-7.

2. Zegers-Hochschild F, Adamson D, Gosta Nygren K, Mouzon J de, Sullivan E, Ishihara O, et al; O-210 Is world IVF going in one direction? Publicado en: Abstracts of the 27th Annual Meeting of the European Society of Human Reproduction and Embryology, Stockholm, Sweden, 3-6 July 2011; Hum Reprod 2011; 26 (1): i84-i85.

3. Boivin J, Bunting L, Collins JA, Nygren KG. International estimates of infertility prevalence and treatment-seeking: potential need and demand for infertility medical care. Hum Reprod 2007; 22 (6): 1506-12.

4. Greil AL, Slauson-Blevins K, McQuillan J. The experience of infertility: a review of recent literature. Sociology of Health \& Illness 2010; 32 (1): 140-62.

5. Van Balen F, Inhorn M. Introduction. Interpreting infertility: A view from the social sciences. En Inhorn M, Van Balen F, editores. Infertility around the Globe: New Thinking on Childlessness, Gender, and Reproductive Technologies. University of California Press; 2002. p. 3-33.

6. Adamson GD, Mouzon J de, Ishihara O, Mansour R, Nygren KG, Sullivan E, et al. International Committee for Monitoring ART Preliminary World Report on ART, Year 2008. 28th Annual Meeting of the European Society for Human Reproduction and Embryology, 2012. Hum Reprod 2012; 27 (suppl 2): ii38-ii39.

7. Zegers-Hochschild F. Descripción y Análisis de la Técnicas de Reproducción Asistida (TRA) como Tratamiento de la Infertilidad. Documento preparado para la Corte Interamericana de Derechos Humanos [Internet]. 2012. Disponible en: http://www.eticayreproduccionhumana. udp.cl/publicaciones/fallo/Documento_tecncio_infertilidad_Problema_salud.pdf.

8. Schwarze J, Crosby J, Zegers-Hochschild F. 20 años de Reproducción Asistida en Chile: 1990-2009. Sociedad Chilena de Medicina Reproductiva; 2012.

9. Hashiloni-Dolev Y, Weiner N. New reproductive technologies, genetic counselling and the standing of the fetus: views from Germany and Israel. Sociology of Health \& Illness 2008; 30 (7): 1055-69.

10. Papaharitou S, Nakopoulou E, Moraitou M, Hatzimouratidis K, Hatzichristou D. Reproductive health and midwives: Does occupational status differentiate their attitudes on assisted reproduction technologies from those of the general population? Hum. Reprod 2007; 22 (7): 2033-9.

11. Silva S, Machado H. The construction of meaning by experts and would-be parents in assisted reproductive technology. Sociology of Health \& Illness 2011; 33 (6): 853-68.

12. Stern JE, Cramer CP, Green RM, Garrod A, DeVries KO. Determining access to assisted reproductive technology: reactions of clinic directors to ethically complex case scenarios. Hum Reprod 2003; 18 (6): 1343-52.

13. Baluch B, Fallone A, Kahn J. Gender Differences and Personality Correlates in Attitudes Towards Controversial Aspects of Human Reproduction. Journal of Social Behavior and Personality 1994; 9 (1): 329-34.

14. Constantinidis D, Cook R. Australian perspectives on surrogacy: the influence of cognitions, psychological and demographic characteristics. Hum Reprod 2012; 27 (4): 1080-7.

15. Chliaoutakis JE, Koukouli S, Papadakaki M. Using attitudinal indicators to explain the public's intention to have recourse to gamete donation and surrogacy. Hum Reprod 2002; 17 (11): 2995-3002.

16. Heikkilä K, Länsimies E, Hippeläinen $M$, Heinonen S. A survey of the attitudes of infertile and parous women towards the availability of assisted reproductive technology. BJOG: An International Journal of Obstetrics \& Gynecology 2004; 111 (11): 1229-35.

17. Lui SC, Weaver SM. Attitudes and motives of semen donors and non-donors. Hum Reprod 1996; 11 (9): 2061-6.

18. Stoop D, Nekkebroeck J, Devroey P. A survey on the intentions and attitudes towards oocyte cryopreservation for non-medical reasons among women of reproductive age. Hum Reprod 2011; 26 (3): 655-61.

19. Baluch B, Fallone A, Anderson R, Furnham A, Aghssa MM. Women's attitude to egg donation and sperm donation-a cross cultural study. Psychology 1994; 31 (3-4): 5-8.

20. Bolton V, Golombok S, Cook R, Bish A, Rust J. A comparative study of attitudes towards donor insemination and egg donation in recipients potential donors and the public. Journal of Psychosomatic Obstetrics \& Gynecology 1991; 12 (3): 217-28.

21. Isikoglu M, Senol Y, Berkkanoglu M, Ozgur K, Donmez L, Stones-Abbasi A. Public opinion regarding oocyte donation in Turkey: first data from a secular population among the Islamic world. Hum Reprod 2006; 21 (1): 318-23.

22. Svanberg AS, Lampic C, Bergh T, Lundkvist Ö. Public opinion regarding oocyte donation in Sweden. Hum Reprod 2003; 18 (5): 1107-14.

23. Minai J, Suzuki K, Takeda Y, Hoshi K, Yamagata Z. There are gender differences in attitudes toward surrogacy when information on this technique is provided. Eur J 
Obstet Gynecol Reprod Biol 2007; 132 (2): 193-9.

24. Poote AE, Van den Akker OBA. British women's attitudes to surrogacy. Hum Reprod 2009; 24 (1): 139-45.

25. Lohr SL. Muestreo: diseño y análisis. Primera Edición. México: Thomson; 2000.

26. Cochran W. Técnicas de Muestreo. México: Compañía editorial Continental; 1980.

27. Kovacs GT, Morgan G, Levine M, Mc Crann J. The Australian community overwhelmingly approves IVF to treat subfertility, with increasing support over three decades. Australian and New Zealand Journal of Obstetrics and Gynecology 2012; 52 (3): 302-4.

28. Stoebel-Richter Y, Geue K, Borkenhagen A, Braehler E, Weidner K. What Do You Know about Reproductive Medicine?-Results of a German Representative Survey. PLoS ONE 2012; 7 (12): e50113.
29. Stöbel-Richter Y, Goldschmidt S, Brähler E, Weidner K, Beutel M. Egg donation, surrogate mothering, and cloning: attitudes of men and women in Germany based on a representative survey. Fertil Steril 2009; 92 (1): 124-30.

30. Parks JA. A closer look at reproductive technology and postmenopausal motherhood. CMAJ 1996; 154 (8): 1189-91.

31. Adashi EY, Cohen J, Hamberger L, Jones HW Jr, De Kretser DM, Lunenfeld B, et al. Public perception on infertility and its treatment: an international survey. The Bertarelli Foundation Scientific Board. Hum Reprod 2000; 15 (2): 330-4.

32. Rauprich O, Berns E, Vollmann J. Who should pay for assisted reproductive techniques? Answers from patients, professionals and the general public in Germany. Hum Reprod 2010; 25 (5): 1225-33. 